\title{
Cell transfer and monomial positivity
}

\author{
Thomas Lam • Pavlo Pylyavskyy
}

Received: 13 June 2005 / Accepted: 1 December 2006 /

Published online: 9 January 2007

(C) Springer Science + Business Media, LLC 2007

\begin{abstract}
We give combinatorial proofs that certain families of differences of products of Schur functions are monomial-positive. We show in addition that such monomialpositivity is to be expected of a large class of generating functions with combinatorial definitions similar to Schur functions. These generating functions are defined on posets with labelled Hasse diagrams and include, for example, generating functions of Stanley's $(P, \omega)$-partitions.
\end{abstract}

Keywords Symmetric functions $\cdot$ Monomial positivity $\cdot$ P-partitions

\section{Introduction}

The Schur functions $s_{\lambda}$ form a basis of the ring of symmetric functions $\Lambda$. They have a remarkable number of combinatorial and algebraic properties, and are simultaneously the irreducible characters of $G L(N)$ and representatives of Schubert classes in the cohomology $H^{*}\left(G r_{k n}\right)$ of the Grassmannian; see $[9,13]$. In recent years, a lot of work has gone into studying whether certain expressions of the form

$$
s_{\lambda} s_{\mu}-s_{\nu} s_{\rho}
$$

are expressible as a non-negative linear combination of Schur functions. See for example $[1,2,4,11]$.

T.L. was supported in part by NSF DMS-0600677.

T. Lam

Department of Mathematics, Harvard University, Cambridge, MA, 02138

e-mail: tfylam@math.harvard.edu

P. Pylyavskyy ( )

Department of Mathematics, M.I.T., Cambridge, MA, 02139

e-mail:pasha@mit.edu 
The first aim of this article is to provide a large class of expressions of the form (1) which are monomial-positive, that is, expressible as a non-negative linear combination of monomial symmetric functions. In particular, we show that (1) is monomial-positive when $\lambda=v \vee \rho$ and $\mu=v \wedge \rho$ are the union and intersections of the Young diagrams of $v$ and $\rho$. However, we show in addition that such monomial-positivity is to be expected of many families of generating functions with combinatorial definitions similar to Schur functions, which are generating functions for semistandard Young tableaux.

We define a new combinatorial object called a $\mathbb{T}$-labelled poset and given a $\mathbb{T}$ labelled poset $(P, O)$ we define another combinatorial object which we call a $(P, O)$ tableau. These $(P, O)$-tableaux include as special cases standard Young tableaux, semistandard Young tableaux, shifted tablueax, cylindric tableaux, plane partitions, and Stanley's $(P, \omega)$-partitions. Our main theorem is the cell transfer theorem. It says that for a fixed $\mathbb{T}$-labelled poset $(P, O)$, one obtains many expressions of the form (1) which are monomial-positive, where the Schur functions in (1) are replaced by generating functions for $(P, O)$-tableaux.

We conjecture that our cell-transfer results for Schur functions hold not just for monomial-positivity but also for Schur-positivity. This conjecture is proved in [8]. In another direction, we strengthen the results of the present article in the case of generating functions of $(P, \omega)$-partitions in [7]. In this case, cell transfer is positive in terms of fundamental quasisymmetric functions.

\section{Posets and tableaux}

Let $(P, \leq)$ be a possibly infinite poset. Let $s, t \in P$. We say that $s$ covers $t$ and write $s>t$ if for any $r \in P$ such that $s \geq r \geq t$ we have $r=s$ or $r=t$. The Hasse diagram of a poset $P$ is the graph with vertex set equal to the elements of $P$ and edge set equal to the set of covering relations in $P$. If $Q \subset P$ is a subset of the elements of $P$ then $Q$ has a natural induced subposet structure. If $s, t \in Q$ then $s \leq t$ in $Q$ if and only if $s \leq t$ in $P$. Call a subset $Q \subset P$ connected if the elements in $Q$ induce a connected subgraph in the Hasse diagram of $P$.

An order ideal $I$ of $P$ is an induced subposet of $P$ such that if $s \in I$ and $s \geq t \in P$ then $t \in I$. A subposet $Q \subset P$ is called convex if for any $s, t \in Q$ and $r \in P$ satisfying $s \leq r \leq t$ we have $r \in Q$. Alternatively, a convex subposet is one which is closed under taking intervals. A convex subset $Q$ is determined by specifying two order ideals $J$ and $I$ so that $J \subset I$ and $Q=\{s \in I \mid s \notin J\}$. We write $Q=I / J$. If $s \notin Q$ then we write $s<Q$ if $s<t$ for some $t \in Q$ and similarly for $s>Q$. If $s \in Q$ or $s$ is incomparable with all elements in $Q$ we write $s \sim Q$. Thus for any $s \in P$, exactly one of $s<Q$, $s>Q$ and $s \sim Q$ is true.

Let $\mathbb{P}$ denote the set of positive integers and $\mathbb{Z}$ denote the set of integers. Let $\mathbb{T}$ denote the set of all weakly increasing functions $f: \mathbb{P} \rightarrow \mathbb{Z} \cup\{\infty\}$.

Definition 2.1. A $\mathbb{T}$-labelling $O$ of a poset $P$ is a map $O:\left\{(s, t) \in P^{2} \mid s>t\right\} \rightarrow$ $\mathbb{T}$ labelling each edge $(s, t)$ of the Hasse diagram by a weakly increasing function $O(s, t): \mathbb{P} \rightarrow \mathbb{Z} \cup\{\infty\}$. A $\mathbb{T}$-labelled poset is an an ordered pair $(P, O)$ where $P$ is a poset, and $O$ is a $\mathbb{T}$-labelling of $P$. 


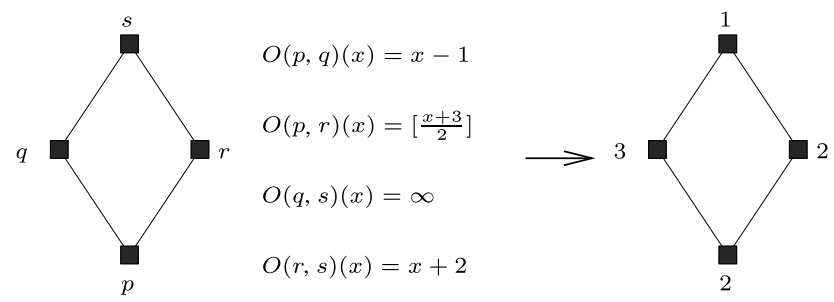

Fig. 1 An example of a $\mathbb{T}$-labelled poset $(P, O)$ and a $(P, O)$-tableau

We shall refer to a $\mathbb{T}$-labelled poset $(P, O)$ as $P$ when no ambiguity arises. If $Q \subset P$ is a convex subposet of $P$ then the covering relations of $Q$ are also covering relations in $P$. Thus a $\mathbb{T}$-labelling $O$ of $P$ naturally induces a $\mathbb{T}$-labelling $\left.O\right|_{Q}$ of $Q$. We denote the resulting $\mathbb{T}$-labelled poset by $(Q, O):=\left(Q,\left.O\right|_{Q}\right)$.

Definition 2.2. A $(P, O)$-tableau is a map $\sigma: P \rightarrow \mathbb{P}$ such that for each covering relation $s \lessdot t$ in $P$ we have

$$
\sigma(s) \leq O(s, t)(\sigma(t))
$$

If $\sigma: P \rightarrow \mathbb{P}$ is any map, then we say that $\sigma$ respects $O$ if $\sigma$ is a $(P, O)$-tableau.

Figure 1 contains an example of a $\mathbb{T}$-labelled poset $(P, O)$ and a corresponding $(P, O)$-tableau.

Denote by $\mathcal{A}(P, O)$ the set of all $(P, O)$-tableaux. If $P$ is finite then one can define the formal power series $K_{P, O}\left(x_{1}, x_{2}, \ldots\right) \in \mathbb{Q}\left[\left[x_{1}, x_{2}, \ldots\right]\right]$ by

$$
K_{P, O}\left(x_{1}, x_{2}, \ldots\right)=\sum_{\sigma \in \mathcal{A}(P, O)} x_{1}^{\# \sigma^{-1}(1)} x_{2}^{\# \sigma^{-1}(2)} \ldots
$$

The composition $\operatorname{wt}(\sigma)=\left(\# \sigma^{-1}(1), \# \sigma^{-1}(2), \ldots\right)$ is called the weight of $\sigma$.

Our $(P, O)$-tableaux can be viewed as a generalization of Stanley's $(P, \omega)$ partitions and also of McNamara's oriented posets; see [10, 13].

Example 2.3. Any Young diagram $P=\lambda$ can be considered as a $\mathbb{T}$-labelled poset $\left(\lambda, O_{\lambda}\right)$. The elements of $\lambda$ are given by the squares of the Young diagram. A square $s \in \lambda$ is less than another square $s^{\prime} \in \lambda$ if and only if $s$ lies (weakly) above and to the left of $s^{\prime}$ when $\lambda$ is drawn in the English notation. The covering relations $\left(s \lessdot s^{\prime}\right)$ are given by pairs of squares sharing an edge.

The labelling $O_{\lambda}$ of the Hasse diagram of $\lambda$ is obtained as follows. An edge $\left(s \lessdot s^{\prime}\right)$ where $s$ and $s^{\prime}$ lie in the same row is labelled with the function $f^{\text {weak }}(x)=x$ and if $s$ and $s^{\prime}$ lie in the same column, the edge is labelled with the function $f^{\text {strict }}(x)=x-1$. $\mathrm{A}\left(\lambda, O_{\lambda}\right)$-tableau is then just a semistandard Young tableaux and we have the equality

$$
K_{\lambda, o_{\lambda}}\left(x_{1}, x_{2}, \ldots\right)=s_{\lambda}\left(x_{1}, x_{2}, \ldots\right)
$$

where $s_{\lambda}\left(x_{1}, x_{2}, \ldots\right)$ is the Schur function labelled by $\lambda$ (see Section 4 ). 
More generally, suppose $\lambda$ and $\mu$ are two partitions satisfying $\mu \subset \lambda$. The skew shape $\lambda / \mu$ can be considered a $\mathbb{T}$-labelled poset, and in this way we obtain the skew Schur functions.

Example 2.4. Another interesting example is given by cylindric tableaux and cylindric Schur functions. Let $1 \leq k<n$ be two positive integers. Let $\mathcal{C}_{k, n}$ be the quotient of $\mathbb{Z}^{2}$ given by

$$
\mathcal{C}_{k, n}=\mathbb{Z}^{2} /(k-n, k) \mathbb{Z}
$$

In other words, the integer points $(a, b)$ and $(a+k-n, b+k)$ are identified in $\mathcal{C}_{k, n}$. We can give $\mathcal{C}_{k, n}$ the structure of a poset by the generating relations $(i, j) \lessdot(i+1, j)$ and $(i, j) \lessdot(i, j+1)$. We give $\mathcal{C}_{k, n}$ a T-labelling $O$ by labelling the edges $(i, j) \lessdot$ $(i+1, j)$ with the function $f^{\text {weak }}(x)=x$ and the edges $(i, j) \lessdot(i, j+1)$ with the function $f^{\text {strict }}(x)=x-1$. A finite convex subposet $P$ of $\mathcal{C}_{k, n}$ is known as a cylindric skew shape; see $[5,10,12]$. The $(P, O)$-tableau are known as semistandard cylindric tableaux of shape $P$ and the generating function $K_{P, O}\left(x_{1}, x_{2}, \ldots\right)$ is the cylindric Schur function defined in $[3,12]$.

Example 2.5. Let $N$ be the number of elements in a poset $P$, and let $\omega: P \longrightarrow[N]$ be a bijective labelling of elements of $P$ with numbers from 1 to $N$. Recall that a $(P, \omega)$ partition (see [13]) is a map $\sigma: P \longrightarrow \mathbb{P}$ such that $s \leq t$ in $P$ implies $\sigma(s) \leq \sigma(t)$, while if in addition $\omega(s)>\omega(t)$ then $\sigma(s)<\sigma(t)$. Label now each edge $(s, t)$ of the Hasse diagram of $P$ with $f^{\text {weak }}$ or $f^{\text {strict }}$, depending on whether $\omega(s) \leq \omega(t)$ or $\omega(s)>\omega(t)$ correspondingly. It is not hard to see that for this labelling $O$ the $(P, O)$-tableaux are exactly the $(P, \omega)$-partitions. Similarly, if we allow any labelling of the edges of $P$ with $f^{\text {weak }}$ and $f^{\text {strict }}$, we get the oriented posets of McNamara; see [10].

\section{The cell transfer theorem}

A generating function $f \in \mathbb{Q}\left[\left[x_{1}, x_{2}, \ldots\right]\right]$ is monomial-positive if all coefficients in its expansion into monomials are non-negative. If $f$ is actually a symmetric function then this is equivalent to $f$ being a non-negative linear combination of monomial symmetric functions.

Let $(P, O)$ be a $\mathbb{T}$-labelled poset. Let $Q$ and $R$ be two finite convex subposets of $P$. The subset $Q \cap R$ is also a convex subposet. Define two more subposets $Q \wedge R$ and $Q \vee R$ by

$$
Q \wedge R=\{s \in R \mid s<Q\} \cup\{s \in Q \mid s \sim R \text { or } s<R\}
$$

and

$$
Q \vee R=\{s \in Q \mid s>R\} \cup\{s \in R \mid s \sim Q \text { or } s>Q\}
$$

Observe that the operations $\vee, \wedge$ are not commutative, and that $Q \cap R$ is a convex subposet of both $Q \vee R$ and $Q \wedge R$. 

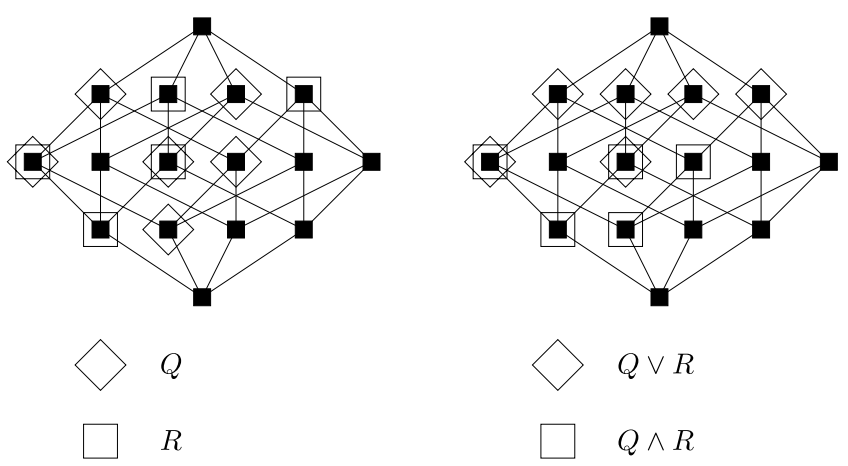

Fig. 2 An example of the operation $(Q, R) \rightarrow(Q \vee R, Q \wedge R)$ inside the boolean lattice $B_{4}$

Example 3.1. Let $P_{n}$ denote the chain with $n+1$ elements labeled $\{0,1, \ldots, n\}$. Then the convex subposets of $P_{n}$ are the intervals $[i, j]$ where $0 \leq i \leq j \leq n$ and $[i, j]$ is isomorphic to the chain with $j-i$ elements. Let $Q=[i, j]$ and $R=\left[i^{\prime}, j^{\prime}\right]$ and assume that $i \leq i^{\prime}$. Then we have the following two cases:

(1) If $j \leq j^{\prime}$ then $Q \wedge R=Q$ and $Q \vee R=R$.

(2) If $j \geq j^{\prime}$ then $Q \wedge R=\left[i, j^{\prime}\right]$ and $Q \vee R=\left[i^{\prime}, j\right]$.

This example leads to interesting combinatorics which we study further in [7].

In Figure 2 an example of the operations $\vee$ and $\wedge$ for two convex subposets of the Boolean lattice $B_{4}$ is given. One can easily check that $Q$ and $R$ are indeed convex and $Q \vee R$ and $Q \wedge R$ are indeed formed according to the rules above.

Recall that cells in a skew Young diagram form a partially ordered set, where each cell is covered by the neighboring cell on the right and the neighboring cell below. Figure 3 gives an example of the operations $\vee$ and $\wedge$ for $Q=(6,5,5,5) /(3,3)$ and $R=(6,6,4,4,4) /(6,1,1,1,1)$, treated as subposets of the poset $\mathbb{N}^{2}$ of boxes in the plane (see Section 4).

Recall that if $A$ and $B$ are sets then $A \backslash B=\{a \in A \mid a \notin B\}$ denotes the set difference.

Lemma 3.2. The subposets $Q \wedge R$ and $Q \vee R$ are both convex subposets of $P$. We have $(Q \wedge R) \cup(Q \vee R)=Q \cup R$ and $(Q \wedge R) \cap(Q \vee R)=Q \cap R$.

Proof: We show that $Q \wedge R$ is convex; the statement for $Q \vee R$ follows similarly. Suppose $s<t$ lie in $Q \wedge R$ and $s<r<t$ for some $r \in P$ but $r \notin Q \wedge R$. Then $t \in Q \wedge R$ implies either $t<Q$ or $t \in Q$. Since $r<t$, we have either $r<Q$ or $r \in Q$.

If $r<Q$ then $s<Q$ and therefore $s \in R$. Thus either $r \in R$ or $r>R$. If $r \in R$ then since $r<Q$, we get $r \in Q \wedge R$, obtaining a contradiction. If $r>R$, then $t>r$ implies $t>R$ which contradicts $t \in Q \wedge R$.

If $r \in Q$ then $r \notin Q \wedge R$ implies $r>R$, and we proceed as above.

The second statement of the lemma is straightforward. 


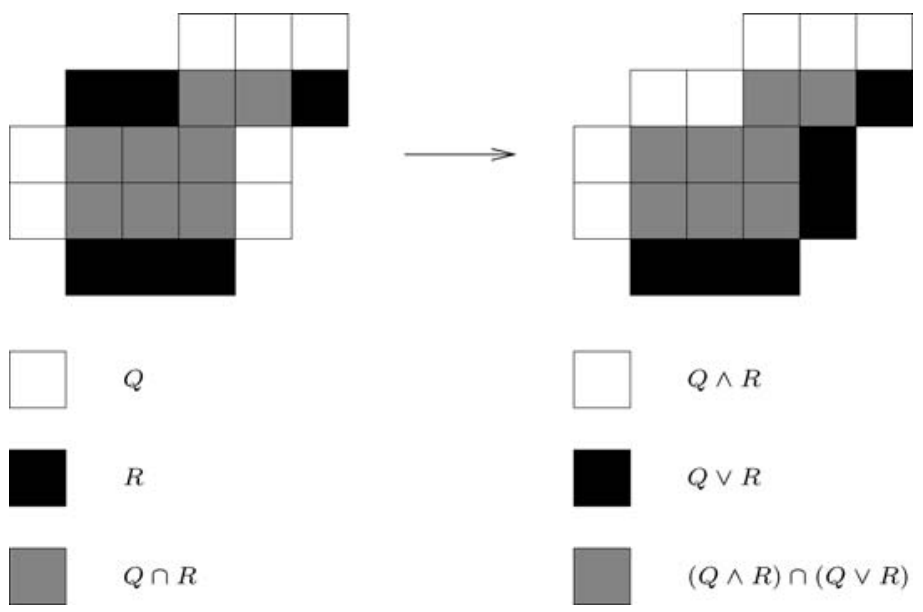

Fig. 3 An example of the operation $(Q, R) \rightarrow(Q \vee R, Q \wedge R)$ for skew Young diagrams

Note that the operations $\wedge$ and $\vee$ are stable so that $(Q \wedge R) \wedge(Q \vee R)=Q \wedge R$ and $(Q \wedge R) \vee(Q \vee R)=Q \vee R$.

Let $\omega$ be a $(Q, O)$-tableau and $\sigma$ be an $(R, O)$-tableau. We now describe how to construct a $(Q \wedge R, O)$-tableau $\omega \wedge \sigma$ and a $(Q \vee R, O)$-tableau $\omega \vee \sigma$. Define a subset of $Q \cap R$, depending on $\omega$ and $\sigma$, by

$$
(Q \cap R)^{+}=\{x \in Q \cap R \mid \omega(x)<\sigma(x)\} .
$$

We give $(Q \cap R)^{+}$the structure of a graph by inducing from the Hasse diagram of $Q \cap R$.

Let $\operatorname{bd}(R)=\{x \in Q \cap R \mid x>y$ for some $y \in R \backslash Q\}$ be the "lower boundary" of $Q \cap R$ which touches elements in $R$. Let bd $(R)^{+} \subset(Q \cap R)^{+}$be the union of the connected components of $(Q \cap R)^{+}$which contain an element of bd $(R)$. Similarly, let $\operatorname{bd}(Q)=\{x \in Q \cap R \mid x \lessdot y$ for some $y \in Q \backslash R\}$ be the "upper boundary" of $Q \cap R$ which touches elements in $Q$. Let $\operatorname{bd}(Q)^{+} \subset(Q \cap R)^{+}$be the union of the connected components of $(Q \cap R)^{+}$which contain an element of bd $(Q)$. The elements in $\operatorname{bd}(Q)^{+} \cup \operatorname{bd}(R)^{+}$are amongst the cells that we might "transfer".

Let $S \subset Q \cap R$. Define $(\omega \wedge \sigma)_{S}: Q \wedge R \rightarrow \mathbb{P}$ by

$$
(\omega \wedge \sigma)_{S}(x)= \begin{cases}\sigma(x) & \text { if } x \in R \backslash Q \text { or } x \in S \\ \omega(x) & \text { otherwise }\end{cases}
$$

Similarly, define $(\omega \vee \sigma)_{S}: Q \vee R \rightarrow \mathbb{P}$ by

$$
(\omega \vee \sigma)_{S}(x)= \begin{cases}\omega(x) & \text { if } x \in Q \backslash R \text { or } x \in S, \\ \sigma(x) & \text { otherwise }\end{cases}
$$

One checks directly that $\operatorname{wt}(\sigma)+\operatorname{wt}(\omega)=\operatorname{wt}\left((\omega \wedge \sigma)_{S}\right)+\operatorname{wt}\left((\omega \vee \sigma)_{S}\right)$. 
Proposition 3.3. Let $(P, O)$ be a $\mathbb{T}$-labelled poset, $Q$ and $R$ be convex subposets of $P$, and $\omega$ and $\sigma$ be $a(Q, O)$-tableau and an $(R, O)$-tableau respectively. Let $S^{*}:=\operatorname{bd}(Q)^{+} \cup \operatorname{bd}(R)^{+}$. Then both $(\omega \wedge \sigma)_{S^{*}}$ and $(\omega \vee \sigma)_{S^{*}}$ respect $O$.

Proof: We check this for $(\omega \wedge \sigma)_{S^{*}}$ and the claim for $(\omega \vee \sigma)_{S^{*}}$ follows from symmetry. Let $s \lessdot t$ be a covering relation in $Q \wedge R$. Since $\sigma$ and $\omega$ are assumed to respect $O$, we need only check the conditions when $(\omega \wedge \sigma)_{S^{*}}(s)=\omega(s)(\neq$ $\sigma(s))$ and $(\omega \wedge \sigma)_{S^{*}}(t)=\sigma(t)(\neq \omega(t))$; or when $(\omega \wedge \sigma)_{S^{*}}(s)=\sigma(s)(\neq \omega(s))$ and $(\omega \wedge \sigma)_{S^{*}}(t)=\omega(t)(\neq \sigma(t))$.

In the first case, we must have $s \in Q$ and $t \in R$. If $t \in R$ but $t \notin Q$ then by the definition of $Q \wedge R$ we must have $t<Q$ and so $t<t^{\prime}$ for some $t^{\prime} \in Q$. This is impossible since $Q$ is convex. Thus $t \in Q \cap R$ and so $t \in S^{*}$. We compute that $\omega(s) \leq$ $O(s, t)(\omega(t)) \leq O(s, t)(\sigma(t))$ since $\omega(t)<\sigma(t)$ and $O(s, t)$ is weakly increasing.

In the second case, we must have $s \in R$ and $t \in Q$. By the definition of $Q \wedge R$ we must have $t \in R$ as well. So $t \in Q \cap R$ but $t \notin S^{*}$ which means that $\omega(t)>\sigma(t)$. Thus $\sigma(s) \leq O(s, t)(\sigma(t)) \leq O(s, t)(\omega(t))$ and $(\omega \wedge \sigma)_{S^{*}}$ respects $O$ here.

For each $(\omega, \sigma)$, we say a subset $S \subseteq S^{*}$ is transferrable if both $(\omega \wedge \sigma)_{S}$ and $(\omega \vee \sigma)_{S}$ respect $O$.

Lemma 3.4. If $S^{\prime}$ and $S^{\prime \prime}$ are both transferrable then so is $S^{\prime} \cap S^{\prime \prime}$.

Proof: Let $s \lessdot t$ be a covering relation of $Q \cup R$. Then the pair $\left((\omega \wedge \sigma)_{S^{\prime} \cap S^{\prime \prime}}(s),(\omega \wedge\right.$ $\left.\sigma)_{S^{\prime} \cap S^{\prime \prime}}(t)\right)$ coincides with one of the four pairs $(\omega(s), \omega(t)),(\sigma(s), \sigma(t)),((\omega \wedge$ $\left.\sigma)_{S^{\prime}}(s),(\omega \wedge \sigma)_{S^{\prime}}(t)\right)$ or $\left((\omega \wedge \sigma)_{S^{\prime \prime}}(s),(\omega \wedge \sigma)_{S^{\prime \prime}}(t)\right)$, depending on the memberships and non-memberships of $s, t$ in $S^{\prime}, S^{\prime \prime}$. Since all these pairs are compatible with $O$, so is the pair $\left((\omega \wedge \sigma)_{S^{\prime} \cap S^{\prime \prime}}(s),(\omega \wedge \sigma)_{S^{\prime} \cap S^{\prime \prime}}(t)\right)$. The same argument applies for $\left((\omega \vee \sigma)_{S^{\prime} \cap S^{\prime \prime}}(s),(\omega \vee \sigma)_{S^{\prime} \cap S^{\prime \prime}}(t)\right)$.

Lemma 3.4 implies that there exists a unique smallest transferrable subset $S^{\diamond} \subseteq S^{*}$. The set $S^{\diamond}$ is the key subset used in the proof of the Cell Transfer Theorem below. It is the set of "transferred cells".

Define $\eta: \mathcal{A}(Q, O) \times \mathcal{A}(R, O) \rightarrow \mathcal{A}(Q \wedge R, O) \times \mathcal{A}(Q \vee R, O)$ by

$$
(\omega, \sigma) \longmapsto\left((\omega \wedge \sigma)_{S^{\diamond}},(\omega \vee \sigma)_{S^{\diamond}}\right)
$$

Note that $S^{\diamond}$ depends on $\omega$ and $\sigma$, though we have suppressed the dependence from the notation.

We call the map $\eta$ the cell transfer procedure. This name comes from our motivating example, where the elements of the poset are the cells of a Young diagram $\lambda$. For convenience, in the following proof, we call elements of any poset $P$ cells. We say that a cell $s \in P$ is transferred if $s \in S^{\diamond}$.

Lemma 3.5. The map $\eta$ is injective. 
Proof: Given $(\alpha, \beta) \in \eta(\mathcal{A}(Q, O) \times \mathcal{A}(R, O))$, we show how to recover $\omega$ and $\sigma$. As before, for a subset $S \subset Q \cap R$, define $\omega_{S}=\omega(\alpha, \beta)_{S}: Q \rightarrow \mathbb{P}$ by

$$
\omega_{S}(x)= \begin{cases}\beta(x) & \text { if } x \in(Q \backslash R) \cap(Q \vee R) \text { or } x \in S, \\ \alpha(x) & \text { otherwise. }\end{cases}
$$

And define $\sigma_{S}=\sigma(\alpha, \beta)_{S}: R \rightarrow \mathbb{P}$ by

$$
\sigma_{S}(x)= \begin{cases}\alpha(x) & \text { if } x \in(R \backslash Q) \cap(Q \wedge R) \text { or } x \in S, \\ \beta(x) & \text { otherwise }\end{cases}
$$

Note that if $\left.(\alpha, \beta)=\left((\omega \wedge \sigma)_{S^{\diamond}},(\omega \vee \sigma)_{S^{\diamond}}\right)\right)$ then $\omega=\omega_{S^{\diamond}}$ and $\sigma=\sigma_{S^{\diamond}}$. Let $S^{\square} \subset$ $Q \cap R$ be the unique smallest subset such that $\omega_{S} \square$ and $\sigma_{S} \square$ both respect $O$. Since we have assumed that $(\alpha, \beta) \in \eta(\mathcal{A}(Q, O) \times \mathcal{A}(R, O))$, such a $S^{\square}$ must exist. (As before the intersection of two transferrable subsets with respect to $(\alpha, \beta)$ is transferrable.)

We now show that if $\left.(\alpha, \beta)=\left((\omega \wedge \sigma)_{S^{\diamond}},(\omega \vee \sigma)_{S^{\diamond}}\right)\right)$ then $S^{\square}=S^{\diamond}$. We know that $S^{\square} \subset S^{\diamond}$ from the previous paragraph. Let $C \subset S^{\diamond} \backslash S^{\square}$ be a connected component of $S^{\diamond} \backslash S^{\square}$, viewed as an induced subgraph of the Hasse diagram of $P$. We claim that $S^{\diamond} \backslash C$ is a transferrable set for $(\omega, \sigma)$; this means that changing $\left.\alpha\right|_{C}$ to $\left.\omega\right|_{C}$ and $\left.\beta\right|_{C}$ to $\left.\sigma\right|_{C}$ gives a pair in $\mathcal{A}(Q \wedge R, O) \times \mathcal{A}(Q \vee R, O)$. Suppose first that $c \in C$ and $s \in S^{\square}$ is so that $c \lessdot s$. By the definition of $S^{\square}$, we must have $\alpha(c) \leq O(c, s)(\beta(s))$ and $\beta(c) \leq O(c, s)(\alpha(s))$. Now suppose that $c \in C$ and $s \in Q \backslash R$ such that $c \lessdot s$. Then we must have $O(c, s)(\omega(s))=O(c, s)(\beta(s)) \geq \alpha(c)=\sigma(c)$. Similar conclusions hold for $c \gg s$. Thus we have checked that $S^{\diamond} \backslash C$ is a transferrable set for $(\omega, \sigma)$, which is impossible by definition of $S^{\diamond}$ : it is the minimal set with this property. Therefore the set $S^{\diamond} \backslash S^{\square}$ is empty and thus $S^{\diamond}=S^{\square}$.

Thus the map $\mu: \eta(\mathcal{A}(Q, O) \times \mathcal{A}(R, O)) \rightarrow \mathcal{A}(Q, O) \times \mathcal{A}(R, O)$ given by

$$
\mu:(\alpha, \beta) \longmapsto\left(\omega(\alpha, \beta)_{S^{\square}}, \sigma(\alpha, \beta)_{S^{\square}}\right)
$$

is inverse to $\eta$. This shows that the map $\left.(\omega, \sigma) \mapsto\left((\omega \wedge \sigma)_{S^{\diamond}},(\omega \vee \sigma)_{S^{\diamond}}\right)\right)$ is injective, completing the proof.

We say that a map between pairs of tableaux weight-preserving if the multiset of their values over all $s \in P$ is not changed by the map.

Theorem 3.6 (Cell Transfer Theorem). The difference

$$
K_{Q \wedge R, O} K_{Q \vee R, O}-K_{Q, O} K_{R, O}
$$

is monomial-positive.

Proof: The map

$$
\eta: \mathcal{A}(Q, O) \times \mathcal{A}(R, O) \longrightarrow \mathcal{A}(Q \wedge R, O) \times \mathcal{A}(Q \vee R, O)
$$



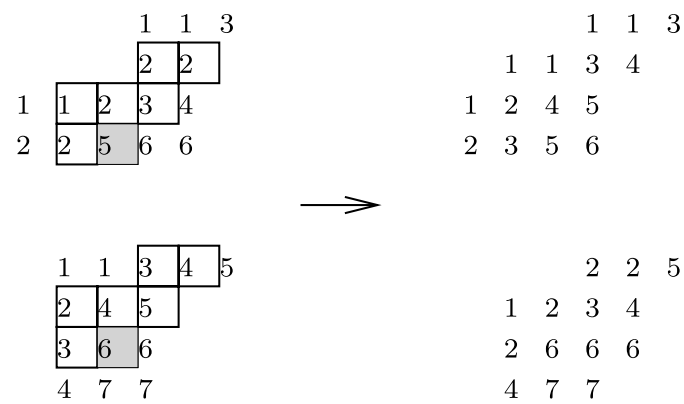

$\begin{array}{lllll} & & 2 & 2 & 5 \\ 1 & 2 & 3 & 4 & \\ 2 & 6 & 6 & 6 & \\ 4 & 7 & 7 & & \end{array}$

Fig. 4 An example of the map $\eta$ applied to a pair of semistandard Young tableaux. The cells in $S^{*}$ are marked, and the unique cell in $S^{*} / S^{\diamond}$ is shaded

defined above is weight-preserving. Indeed, for each element $s \in P \cup Q$ we have $\{\omega(s), \sigma(s)\}=\left\{(\omega \wedge \sigma)_{S^{\diamond}}(s),(\omega \vee \sigma)_{S^{\diamond}}(s)\right\}$ as multisets, where the value of a tableau is zero outside of its range of definition. Then since the map $\eta$ is injective and since $K_{Q \wedge R, O} K_{Q \vee R, O}$ and $K_{Q, O} K_{R, O}$ are the generating functions of common weights of pairs of the tableaux of corresponding shapes, the statement follows.

In Figure 4 an example of the cell transfer injection $\eta$ is given for a pair of tableaux with the shapes $Q=(6,5,5,5) /(3,3)$ and $R=(6,6,4,4,4) /(6,1,1,1,1)$ that were shown in Figure 3. Note that there is one cell contained in $S^{*}$ but not $S^{\diamond}$ : the cell labeled 5 in $Q$ and 6 in $R$.

Note that $(\omega, \sigma) \mapsto\left((\omega \wedge \sigma)_{S^{*}},(\omega \vee \sigma)_{S^{*}}\right)$ also defines a weight-preserving map $\eta^{*}: \mathcal{A}(Q, O) \times \mathcal{A}(R, O) \rightarrow \mathcal{A}(Q \wedge R, O) \times \mathcal{A}(Q \vee R, O)$. Unfortunately, $\eta^{*}$ is not always injective.

Suppose $P$ is a locally-finite poset with a unique minimal element. Let $J(P)$ be the lattice of finite order ideals of $P$; see [13]. If $I, J \in J(P)$ then the subposets $I \wedge J$ and $I \vee J$ of $P$ defined in (2) and (3) are finite order ideals of $P$ and agree with the meet $\wedge J(P)$ and join $\vee_{J(P)}$ of $I$ and $J$ respectively within $J(P)$. In this case the operations $\vee$ and $\wedge$ are commutative.

Now let $P$ be any poset. By defining $Q \wedge^{\prime} R=\{s \in R \mid s<Q\} \cup\{s \in Q \mid s \in$ $R$ or $s<R\}$ and $Q \vee^{\prime} R=\{s \in Q \mid s \sim R$ or $s>R\} \cup\{s \in R \mid s \sim Q$ or $s>Q\}$, the order ideals $I \wedge^{\prime} J=I \wedge_{J(P)} J$ and $I \vee^{\prime} J=I \vee_{J(P)} J$ agree with the meet and join in $J(P)$ even when $P$ does not contain a minimal element.

Proposition 3.7. Let $P$ be a locally-finite poset, let $I, J$ be elements of $J(P)$, and let $O$ be a $\mathbb{T}$-labeling of $P$. Then the generating function

$$
K_{I \wedge_{J(P)} J, O} K_{I \vee_{J(P)} J, O}-K_{I, O} K_{J, O}
$$

is monomial-positive.

Proof: Let $(\omega, \sigma) \in \mathcal{A}(Q, O) \times \mathcal{A}(R, O)$. Replacing $Q \wedge R$ by $Q \wedge^{\prime} R$ and $Q \vee R$ by $Q \vee^{\prime} R$ in (4) and (5) we can define $\left(\omega \vee^{\prime} \sigma\right)_{S}$ and $\left(\omega \wedge^{\prime} \sigma\right)_{S}$. 
The conclusion of Proposition 3.3 holds with $\wedge$ and $\vee$ replaced by $\wedge^{\prime}$ and $\vee^{\prime}$. This is because the set $C$ of elements of $Q \wedge R$ not belonging to $Q \wedge^{\prime} R$ are exactly the elements $s \in Q$ which are incomparable with elements of $R$. These elements belong instead to $Q \vee^{\prime} R$. Since the cells in $C$ are incomparable with elements of $R$, they are in particular never compared with the elements of $Q \cap R$ in the proof of Proposition 3.3. Thus to show that $\left(\omega \vee^{\prime} \sigma\right)_{S^{*}}$ and $\left(\omega \wedge^{\prime} \sigma\right)_{S^{*}}$ respect $O$ the same set of inequalities needs to be verified as in the original Proposition 3.3.

Lemma 3.4 also holds with a verbatim proof if we define $S \subset S^{*}$ to be transferrable if both $\left(\omega \vee^{\prime} \sigma\right)_{S}$ and $\left(\omega \wedge^{\prime} \sigma\right)_{S}$ respect $O$.

Finally, using the same definition (following Lemma 3.4) of the set $S^{\diamond}$, we can obtain a map $\eta^{\prime}: \mathcal{A}(Q, O) \times \mathcal{A}(R, O) \rightarrow \mathcal{A}\left(Q \wedge^{\prime} R, O\right) \times \mathcal{A}\left(Q \vee^{\prime} R, O\right)$ analogous to $\eta$. By the modified versions of Proposition 3.3 and Lemma 3.4 the image of $\eta^{\prime}$ consists of pairs of $O$-compatible labelings. The map $\eta^{\prime}$ is also injective: the proof of Lemma 3.5 remains valid since the cells in $C$ are incomparable with the elements in $S^{\diamond}$, and the inequalities used in the proof of Lemma 3.5 always involve some element of $S^{\diamond}$.

Now the proof of Theorem 3.6 can be modified by replacing $\wedge, \vee$ and $\eta$ with $\wedge^{\prime}$, $\vee^{\prime}$ and $\eta^{\prime}$ to obtain the claimed statement.

\section{Symmetric and Quasisymmetric functions}

We refer to [13] for more details of the material in this section.

Let $n$ be a positive integer. A composition of $n$ is a sequence $\alpha=\left(\alpha_{1}, \alpha_{2}, \ldots, \alpha_{k}\right)$ of positive integers such that $\alpha_{1}+\alpha_{2}+\cdots+\alpha_{k}=n$. We write $|\alpha|=n$. If in addition $\alpha_{1} \geq \alpha_{2} \geq \cdots \geq \alpha_{k}$ then we say that $\alpha$ is a partition of $n$. If $\lambda$ is a partition then $\lambda^{\prime}$ denotes the conjugate partition. Let $l(\lambda)$ denote the number of (non-zero) parts of $\lambda$.

A formal power series $f=f(x) \in \mathbb{Q}\left[\left[x_{1}, x_{2}, \ldots\right]\right]$ with bounded degree is called quasisymmetric if for any $a_{1}, a_{2}, \ldots, a_{k} \in \mathbb{P}$ we have

$$
\left[x_{i_{1}}^{a_{1}} \ldots x_{i_{k}}^{a_{k}}\right] f=\left[x_{j_{1}}^{a_{1}} \ldots x_{j_{k}}^{a_{k}}\right] f
$$

whenever $i_{1}<\cdots<i_{k}$ and $j_{1}<\cdots<j_{k}$. Here $\left[x^{\alpha}\right] f$ denotes the coefficient of $x^{\alpha}$ in $f$. Denote by $\mathcal{Q}$ sym $\subset \mathbb{Q}\left[\left[x_{1}, x_{2}, \ldots\right]\right]$ the space (in fact algebra) of quasisymmetric functions.

Let $\alpha$ be a composition. Then the monomial quasisymmetric function $M_{\alpha}$ is given by

$$
M_{\alpha}=\sum_{i_{1}<\cdots<i_{k}} x_{i_{1}}^{\alpha_{k}} \ldots x_{i_{k}}^{\alpha_{k}}
$$

The set of monomial quasisymmetric functions form a basis of $\mathcal{Q} s y m$. Another basis is given by the fundamental quasi-symmetric functions $L_{\alpha}$ defined as follows:

$$
L_{\alpha}=\sum_{\beta \leq \alpha} M_{\beta},
$$


where for two compositions $\alpha, \beta$ we have $\beta \leq \alpha$ if and only if $\beta$ is a refinement of $\alpha$.

Define (as in Example 2.3) two functions $f^{\text {weak }}, f^{\text {strict }}: \mathbb{P} \rightarrow \mathbb{N} \cup\{\infty\}$ by $f^{\text {weak }}(n)=n$ and $f^{\text {strict }}(n)=n-1$.

Proposition 4.1. Let $(P, O)$ be a finite $\mathbb{T}$-labelled poset. Suppose

$$
O(s, t) \in\left\{f^{\text {weak }}, f^{\text {strict }}\right\}
$$

for each covering relation $s \lessdot t$. Then $K_{P, O}(x)$ is a quasi-symmetric function.

Example 4.2. Let $P_{n}$ denote the chain with $n+1$ elements as in Example 3.1 and let $\alpha=\left(\alpha_{1}, \ldots, \alpha_{k}\right)$ be a composition of $n+1$. Define the labeling $O_{\alpha}$ by setting $O_{\alpha}(i \lessdot i+1)=f^{\text {strict }}$ if $i=\alpha_{1}+\cdots+\alpha_{l}$ for some $1 \leq l \leq k-1$, and $O_{\alpha}(i \lessdot i+$ 1) $=f^{\text {weak }}$ otherwise. Then $K_{P_{n}, O_{\alpha}}=L_{\alpha}$.

A $\mathbb{T}$-labelled poset satisfying the conditions of the proposition is called oriented in [10]. Stanley's $(P, \omega)$-partitions are special cases of $(P, O)$-tableaux, for such posets. If $f \in \mathcal{Q}$ sym then $f$ is $m$-positive if and only if is a non-negative linear combination of the $M_{\alpha}$.

A formal power series $f=f(x) \in \mathbb{Q}\left[\left[x_{1}, x_{2}, \ldots\right]\right]$ with bounded degree is called symmetric if for any $a_{1}, a_{2}, \ldots, a_{k} \in \mathbb{P}$ we have

$$
\left[x_{i_{1}}^{a_{1}} \ldots x_{i_{k}}^{a_{k}}\right] f=\left[x_{j_{1}}^{a_{1}} \ldots x_{j_{k}}^{a_{k}}\right] f
$$

whenever $i_{1}, \ldots, i_{k}$ are all distinct and $j_{1}, \ldots, j_{k}$ are all distinct. Denote by $\Lambda \subset$ $\mathbb{Q}\left[\left[x_{1}, x_{2}, \ldots\right]\right]$ the algebra of symmetric functions. Every symmetric function is quasisymmetric.

Given $\lambda=\left(\lambda_{1}, \lambda_{2}, \ldots\right)$, the monomial symmetric functions $m_{\lambda}$ is given by

$$
m_{\lambda}(x)=\sum_{\alpha} x_{1}^{\alpha_{1}} \ldots x_{k}^{\alpha_{k}}
$$

where the sum is over all distinct permutations $\alpha$ of the entries of the (infinite) vector $\left(\lambda_{1}, \lambda_{2}, \ldots\right)$. As $\lambda$ ranges over all partitions, the $m_{\lambda}$ form a basis of $\Lambda$. If $f \in \Lambda$ then $f$ is monomial-positive if and only if is a non-negative linear combination of the monomial symmetric functions.

Let $\lambda$ be a partition. Recall that a semistandard Young tableau with shape $\lambda$ is a filling of the squares of the Young diagram of $\lambda$ with positive integers so that the rows are weakly increasing and the columns are strictly increasing. The Schur function $s_{\lambda}\left(x_{1}, x_{2}, \ldots\right)$ is the following generating function:

$$
s_{\lambda}\left(x_{1}, x_{2}, \ldots\right)=\sum_{T} x_{1}^{\# 1 \text { 's in } T} x_{2}^{\# 2 \text { 's in } T} \ldots,
$$

where the summation is over all semistandard Young tableaux of shape $\lambda$. More generally one defines the skew Schur functions $s_{\lambda / \mu}\left(x_{1}, x_{2}, \ldots\right)$ in the same manner. 
The Schur functions $s_{\lambda}$ form a basis of $\Lambda$ as $\lambda$ varies over all partitions. If $f \in \Lambda$ is a non-negative linear combination of Schur functions then we call $f$ Schur-positive.

In the following, we will consider all (skew) shapes as convex subposets of the poset $\mathbb{N}^{2}$ of boxes in the plane with partial order $(i, j) \geq\left(i^{\prime}, j^{\prime}\right)$ if and only if $i \geq i^{\prime}$ and $j \geq j^{\prime}$. Thus the $i$-th row of a shape $\lambda$ has cells with coordinates $\left\{(i, 1),(i, 2), \ldots,\left(i, \lambda_{i}\right)\right\}$. Note that we use the word shape to denote a specific such subposet, which may still have multiple representations of the form $\lambda / \mu$. For example, $(6,5,5,5) /(3,3)$ and $(6,5,5,5,1) /(3,3,1)$ represent the same shape but $(6,5,5,5) /(3,3) \neq(7,6,6,6) /(4,4,1,1)$.

Theorem 4.3. The symmetric function $s_{\mu \wedge \lambda} s_{\mu \vee \lambda}-s_{\mu} s_{\lambda}$ is monomial-positive.

Proof: This follows immediately from Theorem 3.6.

Let $\lambda=\left(\lambda_{1}, \ldots, \lambda_{k}\right), \mu=\left(\mu_{1}, \ldots, \mu_{k}\right), v=\left(v_{1}, \ldots, v_{k}\right)$ and $\rho=\left(\rho_{1}, \ldots, \rho_{k}\right)$ be four partitions such that $\mu \subset \lambda$ and $\rho \subset v$. Define the shapes

$$
\max (\lambda / \mu, v / \rho):=\left(\max \left(\lambda_{1}, \nu_{1}\right), \ldots, \max \left(\lambda_{k}, v_{k}\right)\right) /\left(\max \left(\mu_{1}, \rho_{1}\right), \ldots, \max \left(\mu_{k}, \rho_{k}\right)\right)
$$

and

$$
\min (\lambda / \mu, \nu / \rho):=\left(\min \left(\lambda_{1}, v_{1}\right), \ldots, \min \left(\lambda_{k}, v_{k}\right)\right) /\left(\min \left(\mu_{1}, \rho_{1}\right), \ldots, \min \left(\mu_{k}, \rho_{k}\right)\right)
$$

Note that max and min depend on all four partitions $\lambda, \mu, v, \rho$ and not just the shapes $\lambda / \mu$ and $\nu / \rho$. These shapes $\max (\lambda / \mu, \nu / \rho)$ and $\min (\lambda / \mu, \nu / \rho)$ are nearly but not always the same as $\lambda / \mu \vee \nu / \rho$ and $\lambda / \mu \wedge \nu / \rho$ respectively. This is because we may have $\lambda_{i}=\mu_{i}=a$ for some $i$ and then the shape $\lambda / \mu$ does not depend on the exact value of $a$. However, as one can see from the definitions above, the shapes $\max (\lambda / \mu, \nu / \rho)$ and $\min (\lambda / \mu, v / \rho)$ do depend on the choice of $a$.

Fix four partitions $\lambda, \mu, \nu, \rho$ such that $\mu \subset \lambda$ and $\rho \subset v$. Let $V(\lambda / \mu, v / \rho) \subset$ $(\lambda / \mu \cup \nu / \rho)$ denote the set of cells for which $\min (\lambda / \mu, \nu / \rho)$ and $\lambda / \mu \wedge \nu / \rho$ differ. Here $\lambda / \mu \cup v / \rho$ denotes the set theoretic union of the cells lying in $\lambda / \mu$ and $\nu / \rho$. Clearly each cell $s \in V(\lambda / \mu, v / \rho)$ lies in only one of $\lambda / \mu$ or $\nu / \rho$.

Lemma 4.4. Let $s \in V(\lambda / \mu, v / \rho)$. If $s \in \lambda / \mu$ then $s$ is incomparable to all squares in $\nu / \rho$. If $s \in \nu / \rho$ then $s$ is incomparable to all squares in $\lambda / \mu$.

Proof: Let $V_{i} \subset V(\lambda / \mu, v / \rho)$ denote the set of cells in the $i$-th row for which $(\lambda / \mu \vee$ $v / \rho, \lambda / \mu \wedge v / \rho)$ and $(\max (\lambda / \mu, v / \rho), \min (\lambda / \mu, v / \rho))$ differ. If $V_{i}$ is non-empty then either $\lambda_{i}=\mu_{i}$ or $v_{i}=\rho_{i}$ (but not both).

Without loss of generality we assume that $\lambda_{i}=\mu_{i}=a$ so that $V_{i} \subset v / \rho$. Let the leftmost cell in the lowest non-empty row of $\lambda / \mu$ above row $i$ have coordinates $\left(p, a^{\prime}\right)$ and let the rightmost cell in the highest non-empty row of $\lambda / \mu$ below row $i$ have coordinates $\left(q, a^{\prime \prime}\right)$. Then in particular $a^{\prime \prime} \leq a \leq a^{\prime}$. It is easy to check, case by case, that $S_{i} \subset\left\{\left(i, a^{\prime \prime}+1\right),\left(i, a^{\prime \prime}+1\right), \ldots,\left(i, a^{\prime}\right)\right\}$. These cells are incomparable with any cells in $\lambda / \mu$. 
Theorem 4.5. The symmetric function $s_{\max (\lambda / \mu, \nu / \rho)} s_{\min (\lambda / \mu, \nu / \rho)}-s_{\lambda / \mu} s_{\nu / \rho} \quad$ is monomial-positive.

Proof: We give an injection from the set of pairs $(U, T)$ of semistandard tableaux of shape $(\lambda / \mu, \nu / \rho)$ to the set of pairs $\left(U^{\prime}, T^{\prime}\right)$ of semistandard tableaux with shape $(\max (\lambda / \mu, v / \rho), \min (\lambda / \mu, v / \rho))$. First we apply the map $\eta$ of Theorem 3.6 to $(U, T)$ to obtain a pair $\left(U^{\prime \prime}, T^{\prime \prime}\right)$ of semistandard tableaux of shape $(\lambda / \mu \vee \nu / \rho, \lambda / \mu \wedge \nu / \rho)$.

Now define $U^{\prime}$ by letting it be the unique tableau of shape $\max (\lambda / \mu, \nu / \rho)$ with the same numbers as $U^{\prime \prime}$ in the boxes of $\max (\lambda / \mu, v / \rho) \cap(\lambda / \mu \vee v / \rho)$ and with the same numbers as $T^{\prime \prime}$ in the boxes of $\max (\lambda / \mu, \nu / \rho) \cap V(\lambda / \mu, \nu / \rho)$. Similarly define $T^{\prime}$. This process is reversible so the map $(U, T) \rightarrow\left(U^{\prime}, T^{\prime}\right)$ is injective. We claim that $\left(U^{\prime}, T^{\prime}\right)$ is still semistandard, from which the theorem follows.

The claim follows from Lemma 4.4: if a cell $s \in V(\lambda / \mu, \nu / \rho)$ was originally contained in $\nu / \rho$ (respectively $\lambda / \mu$ ) then there is no cell of $\lambda / \mu$ (respectively $\nu / \rho$ ) adjacent to it. Thus checking the inequalities assuring semistandard-ness for a cell $s \in V$ is trivial: the inequality was satisfied in the semistandard tableau $U$ or $T$.

Conjecture 4.6. The symmetric function $s_{\max (\lambda / \mu, \nu / \rho)} s_{\min (\lambda / \mu, \nu / \rho)}-s_{\lambda / \mu} s_{\nu / \rho}$ is Schurpositive.

This conjecture is proved in joint work [8] with Alex Postnikov. The proof relies ultimately on some deep results in representation theory.

A combinatorial proof of the weaker statement that this same expression is positive in terms of fundamental quasisymmetric functions is given in [7].

\section{Cell transfer as an algorithm}

Let $(P, O)$ be a $\mathbb{T}$-labelled poset. We now give an algorithmic description of cell transfer. Let $Q$ and $R$ be two finite convex subposets of $P$. We construct step-by-step an injection

$$
\eta: \mathcal{A}(Q, O) \times \mathcal{A}(R, O) \longrightarrow \mathcal{A}(Q \wedge R, O) \times \mathcal{A}(Q \vee R, O)
$$

which is weight-preserving. Let $\omega$ be a $(Q, O)$-tableau and $\sigma$ be an $(R, O)$-tableau. Let us recursively define $\bar{\omega}: Q \wedge R \rightarrow \mathbb{P}$ and $\bar{\sigma}: Q \vee R \rightarrow \mathbb{P}$ as follows.

(1) Define $\bar{\omega}: Q \wedge R \rightarrow \mathbb{P}$ and $\bar{\sigma}: Q \vee R \rightarrow \mathbb{P}$ as follows:

$$
\begin{aligned}
& \bar{\omega}(s)= \begin{cases}\omega(s) & \text { if } s \in Q, \\
\sigma(s) & \text { if } s \in R / Q .\end{cases} \\
& \bar{\sigma}(s)= \begin{cases}\sigma(s) & \text { if } s \in R, \\
\omega(s) & \text { if } s \in Q / R .\end{cases}
\end{aligned}
$$

Note that $\bar{\omega}$ and $\bar{\sigma}$ do not necessarily respect $O$. Indeed, the parts of $\omega$ and $\sigma$ which we glued together might not agree with each other, i.e., a covering relation $s \lessdot t$ 
might fail to respect $O$, where the label of one of $s, t$ comes from $\omega$, and that of the other from $\sigma$.

(2) We say that we transfer a cell $s \in Q \cap R$ when we swap the values at $s$ of $\bar{\omega}$ and $\bar{\sigma}$. We say that a cell $s$ in $Q \cap R$ is critical if one of the following condition holds

(a) for some $t \in R$ and $s>t$ we have $O(s, t)(\bar{\omega}(s))<\bar{\omega}(t)$,

(b) for some $t \in Q \cap R$ and $t>s$ we have $O(s, t)(\bar{\sigma}(t))<\bar{\sigma}(s)$,

(c) for some $t \in Q$ and $t>s$ we have $O(s, t)(\bar{\sigma}(t))<\bar{\sigma}(s)$,

(d) for some $t \in Q \cap R$ and $s>t$ we have $O(s, t)(\bar{\omega}(s))<\bar{\omega}(t)$,

and $s$ was not transferred in a previous iteration. We now transfer all critical cells if there are any.

(3) Repeat step (2) until no critical cells are transferred.

Theorem 5.1 (Cell Transfer Algorithm). The algorithm described above terminates in a finite number of steps. The resulting maps $\bar{\omega}$ and $\bar{\sigma}$ are $(P, O)$-tableaux and coincide with $(\omega \wedge \sigma)_{S^{\diamond}}$ and $(\omega \vee \sigma)_{S^{\diamond}}$ defined in the proof of Theorem 3.6.

Proof: As for the first claim, there is a finite number of cells in $Q \cap R$ and each gets transferred at most once, thus the process terminates.

We say that an edge $a \lessdot b$ in the Hasse diagram of $P$ respects $O$ if $\bar{\omega}(a) \leq$ $O(a, b)(\bar{\omega}(b))$ and $\bar{\sigma}(a) \leq O(a, b)(\bar{\sigma}(b))$, whenever these inequalities make sense. Note that a cell $s$ is critical only if the cell $t$ (from the definition of a critical cell) was transferred in previous iteration of step (2), or if it is the first iteration of step (2) and $t$ belongs to $\{s \in R \mid s<Q\}$ or $\{s \in Q \mid s>R\}$ - the parts which were "glued" in step (1). Indeed, if $s, t$ have both not been transferred then $s \lessdot t$ must respect $O$ since $\omega$ and $\sigma$ were $(P, O)$-tableaux to begin with. Similarly, two cells $s \lessdot t$ which have both been transferred must also respect $O$.

We thus see that after the second step every edge between $\{s \in R \mid s<Q\}$ and $Q \cap R$, as well as between $\{s \in Q \mid s>R\}$ and $Q \cap R$ respects $O$. After the algorithm terminates every edge in $Q \cap R$ must respect $O$, since if there exists an edge $s \lessdot t$ which does not then one of $s$ and $t$ must have already been transferred, and the other has not been transferred and thus is critical. This contradicts the termination condition of the algorithm. Therefore, the only possible edges which might fail to respect $O$ are the ones between $\{s \in Q \mid s<R\}$ and $Q \cap R$, and the ones between $\{s \in R \mid s>Q\}$ and $Q \cap R$. However, it is easy to see that during the whole process values of $\bar{\omega}$ on $Q \cap R$ are increasing, and therefore cannot be not large enough for values of $\bar{\omega}$ on $\{s \in Q \mid s<R\}$. Similarly, values of $\bar{\sigma}$ on $Q \cap R$ are decreasing and cannot be too large for values of $\bar{\sigma}$ on $\{s \in R \mid s>Q\}$. Thus, we do obtain two $(P, O)$-tableaux $\bar{\omega}$ and $\bar{\sigma}$.

Let $\bar{S} \subset Q \cap R$ be the set of cells we transferred during the algorithm. The fact that values of $\bar{\omega}$ on $Q \cap R$ increase and the values of $\bar{\sigma}$ on $Q \cap R$ decrease implies that $\bar{S}$ is contained in $S^{*}$ (as defined in the proof of Theorem 3.6). We claim that all transferrable sets contain $\bar{S}$. Indeed, in each iteration we transfer only those cells that must be transferred in order for the result to respect $O$. On the other hand, as shown above the set $\bar{S}$ is transferrable itself. Thus, it is exactly the set $S^{\diamond}$ the minimal transferrable set. This completes the proof of the theorem. 


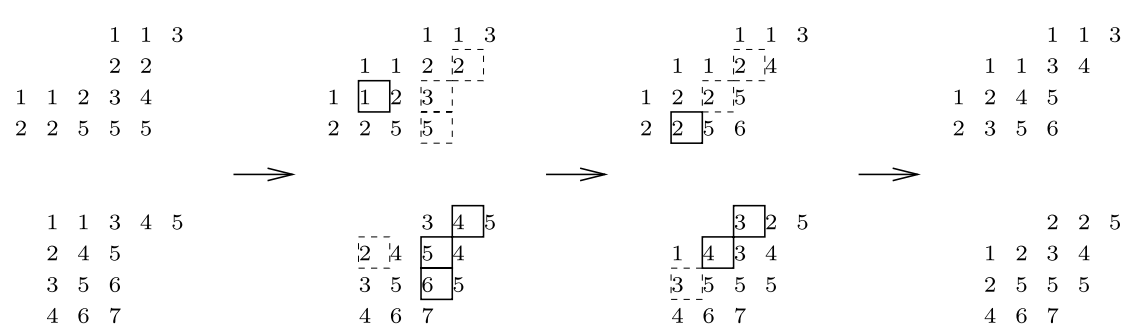

Fig. 5 An example of the cell transfer algorithm applied to a pair of semistandard tableaux. The critical cells are marked with squares

The algorithmic description above provides another way to verify injectivity of $\eta$. Let $\bar{\omega}: Q \wedge R \rightarrow \mathbb{P}$ and $\bar{\sigma}: Q \vee R \rightarrow \mathbb{P}$ be in the image of $\eta$. Then one can define $\operatorname{maps} \omega^{\prime}: Q \rightarrow \mathbb{P}$ and $\sigma^{\prime}: R \rightarrow \mathbb{P}$ by

$$
\begin{aligned}
& \omega^{\prime}(s)= \begin{cases}\bar{\omega}(s) & \text { if } s \in Q \cap(Q \wedge R), \\
\bar{\sigma}(s) & \text { otherwise }\end{cases} \\
& \sigma(s)= \begin{cases}\bar{\sigma}(s) & \text { if } s \in R \cap(Q \vee R), \\
\bar{\omega}(s) & \text { otherwise. }\end{cases}
\end{aligned}
$$

We now iterate step (2) of the cell transfer algorithm with $\omega^{\prime}$ and $\sigma^{\prime}$ replacing $\bar{\omega}$ and $\bar{\sigma}$.

One can verify that for each step the set of transferred cells is identical to the corresponding step of the original algorithm for $\bar{\omega}$ and $\bar{\sigma}$. This produces the inverse of $\eta$.

In Figure 5, we show the step-by-step application of the cell transfer algorithm to a pair of semistandard tableaux $(\omega, \sigma)$.

\section{Final remarks}

The most interesting feature of the cell transfer theorem is that for the case of Schur functions, the theorem holds with monomial-positivity replaced by Schur positivity [8]. Also we show in [7] a similar result holds for generating functions of $(P, \omega)$-partitions with fundamental quasi-symmetric functions replacing Schur functions. We know of no simple combinatorial explanation of these phenomena. A natural question to ask is whether a result similar to Conjecture 4.6 holds for other $\mathbb{T}$-labelled posets $(P, O)$. When $P$ has a minimal element, it seems reasonable to replace Schur-positivity in the conjecture by positivity in the generating functions $\left\{K_{I, O} \mid I\right.$ is an order ideal of $\left.P\right\}$. However, it is not even clear under what conditions the functions $\left\{K_{I, O}\right\}$ might span the space of functions $\left\{K_{Q, O} \mid Q\right.$ is a convex subposet of $\left.P\right\}$ or span the space of differences of products of such functions. $\mathbb{T}$-labelled posets satisfying these weaker requirements would also be worth studying.

Cylindric Schur functions provide another interesting special case. It is conjectured in [6] that the cylindric Schur functions $K_{(P, O)}\left(x_{1}, x_{2}, \cdots\right)$, where $P$ is a cylindric skew 
shape (see Example 2.4) is a positive linear combination of symmetric functions known as affine Schur functions or dual k-Schurfunctions (see also [10]). One might speculate that the differences of products of cylindric Schur functions from Theorem 3.6 are also affine Schur-positive.

Acknowledgments We would like to thank our advisor Richard Stanley, for interesting conversations concerning this problem. We thank the anonymous referee for many helpful suggestions.

\section{References}

1. F. Bergeron and P. McNamara, Some positive differences of products of Schur functions, preprint, 2004; math.CO/0412289.

2. F. Bergeron, R. Biagioli, and M. Rosas, "Inequalities between Littlewood-Richardson Coefficients," $J$. Comb. Th. Ser A, to appear; math. C0/0403541.

3. N. Bergeron and F. Sottile, "Skew Schubert functions and the Pieri formula for flag manifolds, with Nantel Bergeron," Trans. Amer. Math. Soc. 354, (2002), 651-673.

4. S. Fomin, W. Fulton, C.-K. Li, and Y.-T. Poon, "Eigenvalues, singular values, and LittlewoodRichardson coefficients," Amer. J. Math. 127 (2005), 101-127.

5. I. Gessel and C. Krattenthaler, "Cylindric Partitions," Trans. Amer. Math. Soc. 349 (1997), 429-479.

6. T. Lam, "Affine Stanley Symmetric Functions," Amer. J. Math., to appear; math. C0/0501335.

7. T. Lam and P. Pylyavskyy, " $P$-partition products and fundamental quasi-symmetric function positivity," preprint, 2006; math. CO/0609249.

8. T. Lam, A. Postnikov, and P. Pylyavskyy, "Schur positivity and Schur log-concavity," Amer. J. Math., to appear; math. CO/0502446.

9. I. Macdonald, Symmetric Functions and Hall Polynomials, Oxford University Press, 1995.

10. P. McNamara, "Cylindric Skew Schur Functions," Adv. Math. 205(1) (2006), 275-312.

11. A. Okounkov, "Log-Concavity of multiplicities with Applications to Characters of $U(\infty)$," Adv. Math. 127(2) (1997), 258-282.

12. A. Postnikov, "Affine approach to quantum Schubert calculus," Duke Math. J. 128(3) (2005), 473-509.

13. R. Stanley, Enumerative Combinatorics, Vol 2, Cambridge, 1999. 